\title{
Personalized Approach to Tissue and Liquid Biopsy after Failure of First-Line EGFR-TKIs: Is There an Issue When Tissue Is Not the Issue? A Case Series
}

\author{
Daliborka Bursac a, b Bojan Zarića, ${ }^{a} \quad$ Tomi Kovačevića, b Vladimir Stojšića, b \\ Anastasios Vagionas ${ }^{c}$ Ioannis Boukovinas ${ }^{d}$ Kosmas Tsakiridis $^{e}$ \\ Christoforos Kosmidis $^{f}$ Konstantinos Sapalidis ${ }^{f}$ Konstantinos Romanidis 9 \\ Nikolaos Courcoutsakis ${ }^{h}$ Dimitris Matthaios ${ }^{i}$ Paul Zarogoulidis ${ }^{f, j}$ \\ Chrysanthi Sardelik Vanesa Sekerus ${ }^{a, b}$
}

\begin{abstract}
aFaculty of Medicine, University of Novi Sad, Novi Sad, Serbia; 'b Institute for Pulmonary Diseases of Vojvodina, Sremska Kamenica, Serbia; ' Oncology Department, General Hospital of Kavala, Kavala, Greece; 'Oncology Department, "Bioclinic" Private Department, Thessaloniki, Greece; eThoracic Surgery Department, "Interbalkan" European Medical

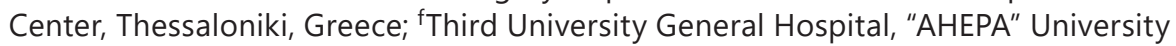
Hospital, Thessaloniki, Greece; 9Second Department of Surgery, General University Hospital of Alexandroupolis, Medical School, Democritus University of Thrace, Alexandroupolis,

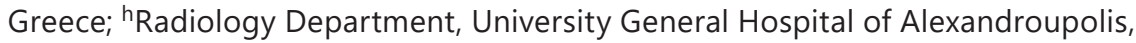
Democritus University of Thrace, Medical School of Health Sciences, Alexandroupolis, Greece; 'Oncology Department, General Hospital of Rhodes, Rhodes, Greece; jPulmonary Department, "Bioclinic" Private Hospital, Thessaloniki, Greece; ${ }^{2}$ Department of Pharmacology and Clinical Pharmacology, School of Medicine, Faculty of Health Sciences, Aristotle University of Thessaloniki, Thessaloniki, Greece
\end{abstract}

\section{Keywords}

T790M mutation · Tissue biopsy $\cdot$ Liquid biopsy $\cdot$ Osimertinib

\begin{abstract}
Traditionally, tissue availability from rebiopsy is a prerequisite for adequate sequencing of epidermal growth factor receptor tyrosine kinase inhibitors (EGFR-TKIs) in therapy for advanced-stage lung cancer. Tissue biopsy truly is the gold standard for genetic analyses, but in some cases, such as with inadequate localization of the lesion or a patient's inadequate performance status, comorbidities, or unwillingness to undergo an invasive procedure, liquid biopsy-based ctDNA analysis can be a noninvasive alternative approach. However, in some cases the gold standard might not shine that much. It is known that tumor heterogeneity or an inadequate amount of tissue might significantly interfere with the results of testing. In this paper, we present cases of patients with a negative tissue biopsy but a positive liquid biopsy
\end{abstract}


which identified coexisting T790M mutation. These results enabled adequate sequencing and treatment with third-line EGFR-TKIs. Such possibilities stress the need to individualize testing for driver mutations in cases where it is clinically highly indicated.

(C) 2021 The Author(s).

Published by S. Karger AG, Basel

\section{Introduction}

The activating epidermal growth factor receptor (EGFR) mutation predicts sensitivity to first- and second-generation EGFR tyrosine kinase inhibitors (TKIs) such as erlotinib, gefitinib, afatinib, or dacomitinib. These molecular alterations are present in almost $50-60 \%$ of patients with advanced non-small-cell lung cancer (NSCLC) in the Asian populations and about $10-15 \%$ in the Caucasian population $[1,2]$. The overall response rate and progressionfree survival with EGFR-TKIs are superior to those with standard first-line platinum doublet chemotherapy, making this therapy a standard of care. However, tumors acquire resistance after approximately 9-13 months [1]. Substitution of threonine with methionine at amino acid position 790 (T790M) in exon 20 of the EGFR gene is the most important acquired resistance mechanism $[1,3]$. In approximately $50-60 \%$ of cases of resistance, T790M mutation occurs as a secondary mutation, whereas as a primary mutation it only occurs in less than $1 \%$ of cases [3].

Osimertinib is an orally available third-generation TKI efficient in patients with EGFR-TKI sensitization (EGFRm) and T790M mutation-positive advanced NSCLC, with less frequent and less severe gastrointestinal and skin toxicity compared with first- or second-generation and greater penetration through the blood-brain barrier [4].

The basic principle for guidance of treatment after failure of first-line EGFR-TKI treatment is rebiopsy. Tissue biopsy gives the best results, but lack of available tissue for molecular assessment is common in daily practice. The most common problems include an inaccessible location or a small size of the tumor at the time of disease progression, an unacceptable risk of complications with invasive diagnostics, tumor heterogeneity, or patient disagreement with invasive methods [5].

Liquid biopsies based on circulating tumor DNA (ctDNA) analysis have many advantages such as early detection of resistance mutations, surrogate samples for tumor molecular analysis, and the potential of being a dynamic marker for prediction of outcome of the therapy [1].

\section{Patients and Methods}

Patient demographic and clinical data were extracted from the institutional lung cancer registry and patient record database of the Institute for Pulmonary Diseases of Vojvodina. All available pathology slides and formalin-fixed paraffin-embedded tumor tissue specimens from each patient were reviewed by a pathologist to confirm the diagnosis and to select a representative sample containing the most viable tumor cells for molecular diagnostics. Histological and cytological specimens were processed and genomic DNA was isolated using the Cobas ${ }^{\circledR}$ DNA Sample Preparation Kit (Roche Molecular Systems) according to the manufacturer's protocol. Measurement of DNA concentration was performed using the Qubit ${ }^{\circledR}$ dsDNA BR Assay Kit and the Qubit ${ }^{\circledR}$ Fluorometer (Thermo Fisher Scientific). Two milliliters of K2 EDTA blood plasma samples were prepared and circulating cell-free DNA (cfDNA) isolated using the Cobas ${ }^{\circledR}$ cfDNA Sample Preparation Kit according to the manufacturer's instructions (Roche Molecular Systems).

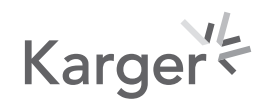


Fig. 1. Chest X-ray in October 2014.

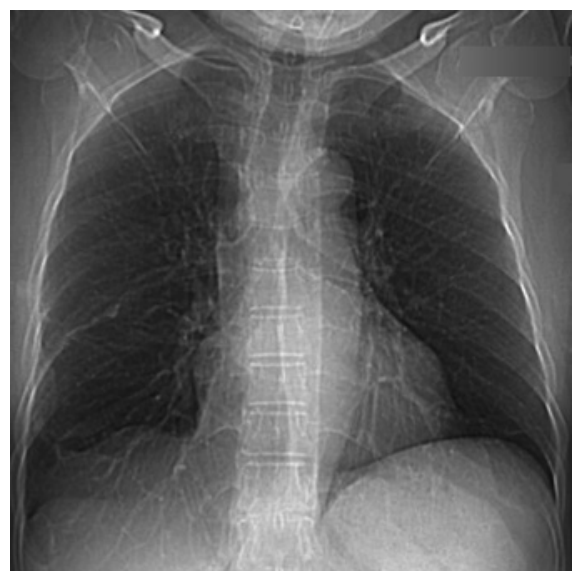

Final procedures included Cobas ${ }^{\circledR}$ EGFR Mutation Test-based PCR amplification and detection of target DNA using complementary primer pairs and oligonucleotide probes labeled with fluorescent dyes (Roche Molecular Systems). The target tissue-derived DNA and plasma-derived cfDNA were amplified and detected on the Cobas ${ }^{\circledR} \mathrm{z} 480$ analyzer. A mutant control and negative control were included in each run to confirm the validity of the run.

VENTANA anti-ALK (D5F3) Rabbit Monoclonal Primary Antibody stained with OptiView DAB Detection and Amplification was used for the detection of ALK protein. The immunohistochemistry (IHC) technique is routine in the institutional pathology laboratory. The Dako PD-L1 IHC clone 22C3 was used for programmed death ligand 1 (PD-L1) testing, and the Tumor Proportion Score (TPS) was used for interpretation of the staining. Both procedures are routine and standardized in the institutional pathology department.

\section{Case Reports}

Case 1

A 66-year-old woman, a nonsmoker with no comorbidities, presented with complaints of dyspnea and dry cough in October 2014. Her hematological and biochemical parameters were within normal limits. A chest X-ray showed right lower lobe infiltration and right pleural effusion (Fig. 1). Computed tomography (CT) of the chest was suggestive of a primary mass lesion in the right lower lobe, $6.5 \mathrm{~cm}$ in diameter with hilar lymph node enlargement. A CT scan of the abdomen showed a metastatic lesion of the left adrenal gland. Pleural fluid cytology was positive for lung adenocarcinoma. The stage of the disease was cT3N1M1b, and her EGFR mutation status was reported as deletion in exon 19. The patient was treated with a first line of a TKI (gefitinib at $250 \mathrm{mg}$ ) for 30 months. A partial radiological response was achieved according to RECIST (Response Evaluation Criteria in Solid Tumors) v.1.1.

In March 2017, radiological progression of the disease was observed, without clinical deterioration. CT scans of the chest and abdomen showed an increased primary tumor in the right lung and metastatic lesions in the left adrenal gland. The patient underwent bronchoscopy for the first time. The endoscopic finding was normal, and histological examination of the transbronchial biopsy (TBB) from the right upper lobe confirmed lung adenocarcinoma. However, EGFR testing of the TBB material was negative. Since there was an insufficient amount of tissue available from the TBB, the multidisciplinary tumor board decided to repeat bronchoscopy for a wider testing panel including PD-L1 testing, which had become available

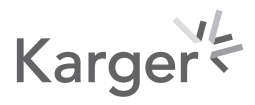




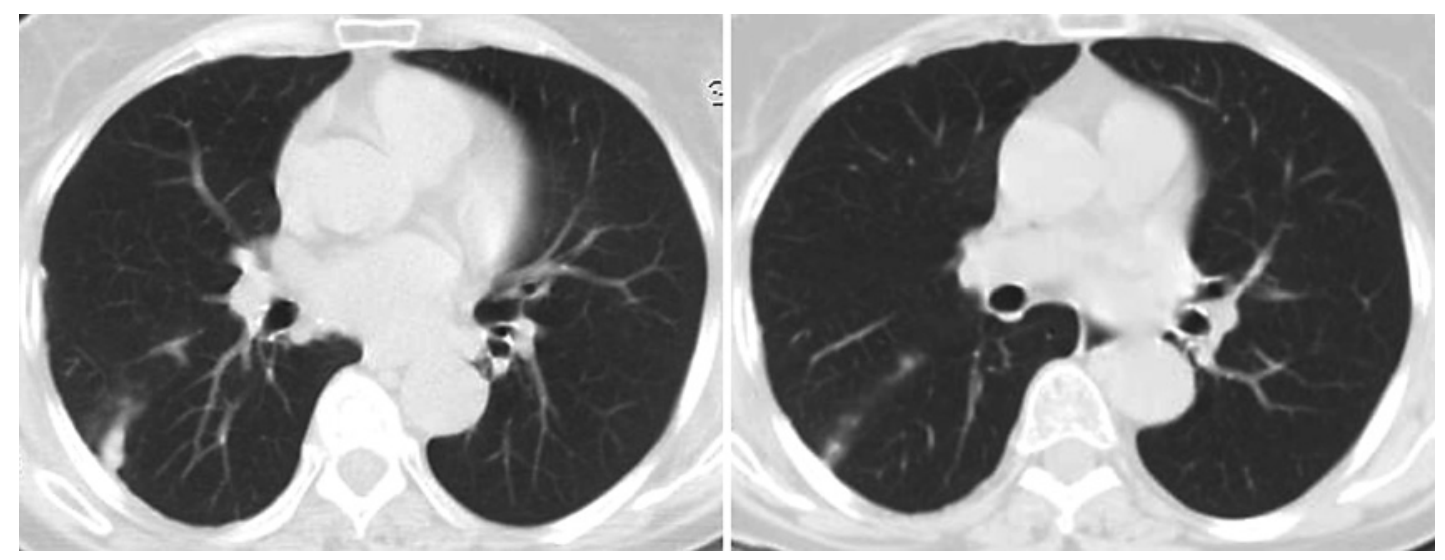

Fig. 2. Chest $\mathrm{CT}$ at the start of treatment with osimertinib and after 3 months.

meanwhile. Once again, an EGFR test was performed on the histology sample, and immunohistochemistry for ALK and PD-L1 was done for the first time. All three tests came out negative. Since the patient had an Eastern Cooperative Oncology Group (ECOG) performance status of 0 , without any symptoms, the patient requested to have a liquid biopsy test performed. Nextgeneration sequencing tests were not available at that time, but liquid biopsy EGFR testing was available in house.

A liquid biopsy was performed, and the result indicated the presence of T790M mutation. The patient started treatment with osimertinib in the second line within a compassionate use program. CT scans of the chest and abdomen showed a partial therapeutic response after 3 months of treatment (Fig. 2), while brain CT results remained normal throughout treatment. The patient is still on osimertinib therapy (total duration: 59 months) without symptoms from the primary tumor and without side effects.

\section{Case 2}

A 68-year-old man with no smoking history and comorbidities presented with exertional dyspnea and cough in April 2017. CT scanning of the chest revealed a nodule $(2.8 \times 1.4 \mathrm{~cm})$ in the right lower lobe, a nodulus in the same lobe, enlarged hilar lymph nodes, and pleural effusion. A cytological examination of the pleural effusion confirmed malignancy, i.e., lung adenocarcinoma. The patient was diagnosed with T3N3M1a stage IV lung adenocarcinoma with positive pleural effusion. An examination of the EGFR mutation status from the pleural effusion confirmed the presence of deletion in exon 19.

Gefitinib therapy ( $250 \mathrm{mg}$ /day taken orally) was chosen as first-line therapy. Within 6 months, the patient experienced a partial remission of the lung disease. A CT scan indicated that the nodule in the right lower lobe was smaller and the pleural effusion was decreased. During gefitinib treatment, the patient had side effects in the form of an increase in liver transaminases, to a mild degree.

After 16 months of follow-up, a CT scan showed that the lesion in the right lower lobe had grown. However, the overall RECIST evaluation was still within the stable disease boundaries. The patient became more symptomatic and tumor board decided to go for re-biopsy even though the was no apparent progression. Bronchoscopy was performed, with endoscopically visible extramural compression. Histological examination of the catheter biopsy and TBB from the right upper lobe confirmed solid type lung adenocarcinoma. Tissue EGFR testing confirmed an old preexisting mutation, a deletion in exon 19. However, due to high symptom

\section{Karger'}



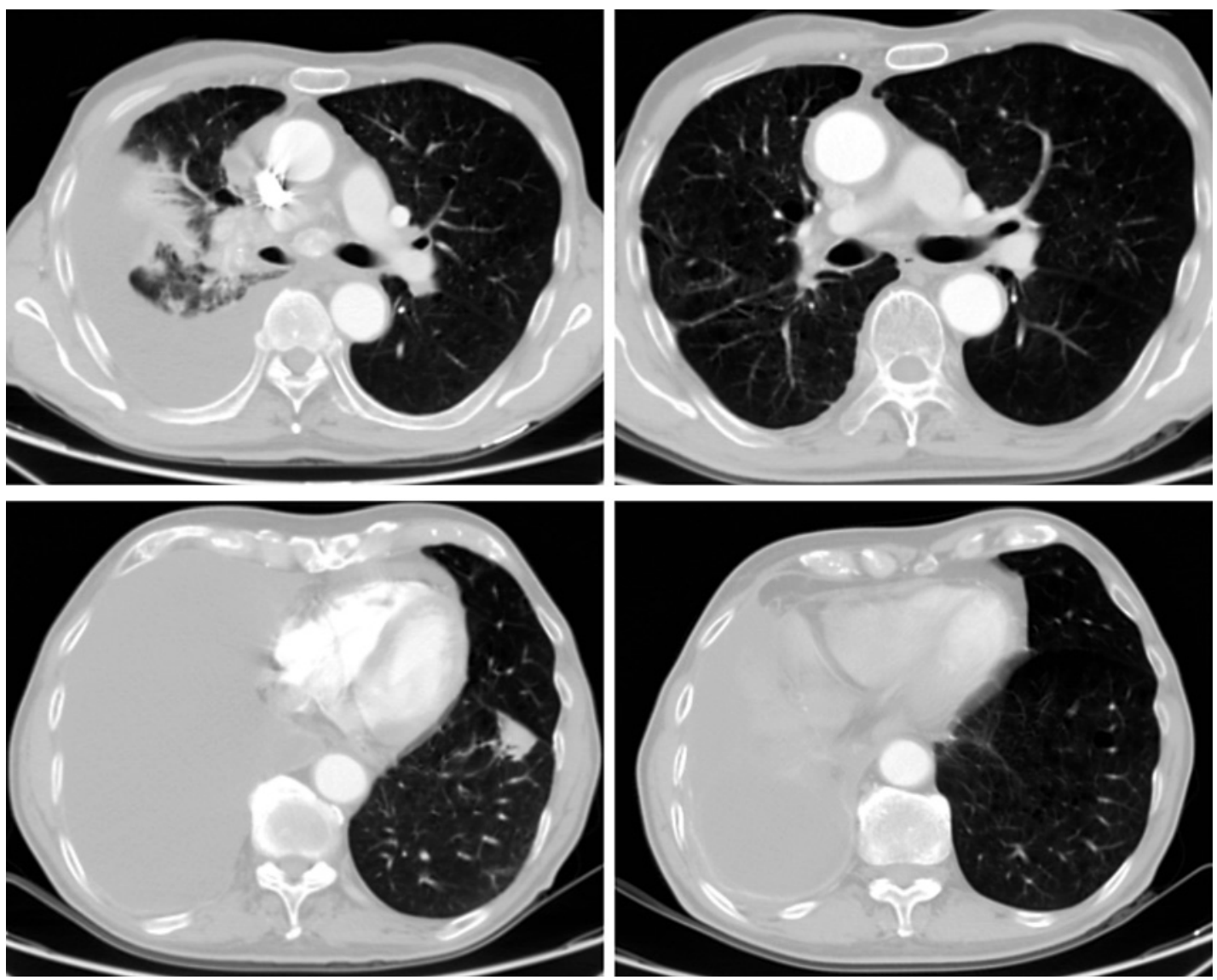

Fig. 3. Chest CT at the start of treatment with osimertinib and after 3 months.

burden the board decides to go for confirmatory liquid biopsy which reveals coexisting deletion 19 with T790M mutation.

The patient underwent pleural drainage and pleurodesis of the right-sided effusion. After control of her symptoms, treatment started with osimertinib ( $80 \mathrm{mg} /$ day). After 3 months, a CT scan showed partial remission of the lung disease (Fig. 3.) Currently, 28 months after the diagnosis, the patient is doing well without any side effects and continues with osimertinib treatment.

\section{Case 3}

In September 2015, a 75-year-old, never-smoking female came to the hospital with cough and chest pain. Clinical examination revealed that she was in good overall condition. She also suffered from arterial hypertension and rheumatoid arthritis. A chest X-ray showed right upper lobe infiltration. CT of the chest showed a tumorous lesion in the right upper lobe, 6.5 $\mathrm{cm}$ in diameter with multiple bilateral pulmonary nodules, but without lymph node enlargement (Fig. 4). A CT scan of the abdomen was without any pathological finding.

Bronchoscopy produced no pathological endoscopic finding, and pathohistological examination of TBB from the right upper lobe confirmed lung adenocarcinoma at stage T4N0M1a. EGFR testing confirmed the existence of L858R mutation in exon 21 and T790M mutation in exon 20. Following confirmation of the EGFR mutation status, the patient received first-line treatment with gefitinib at $250 \mathrm{mg}$. Total treatment with gefitinib was carried out for a period 


\section{Case Reports in Oncology}
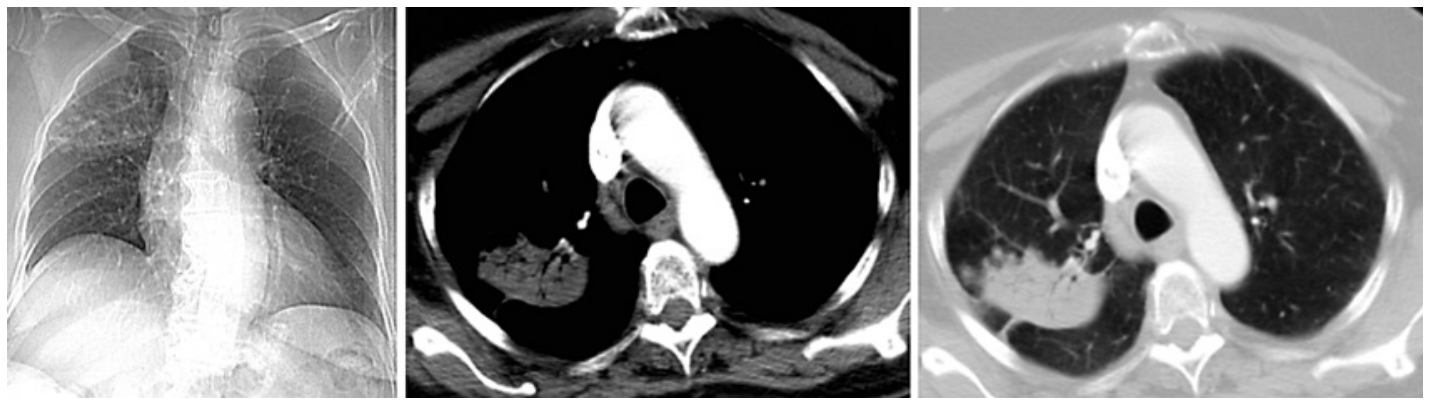

Fig. 4. Chest X-ray and CT at the time of diagnosis.
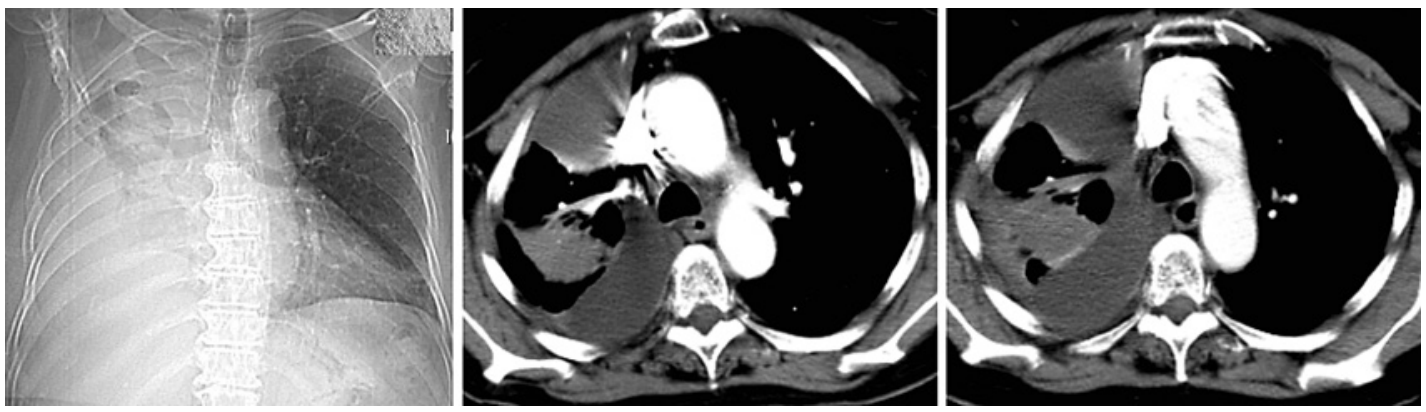

Fig. 5. Chest X-ray and CT scan at the time of progression to gefitinib.
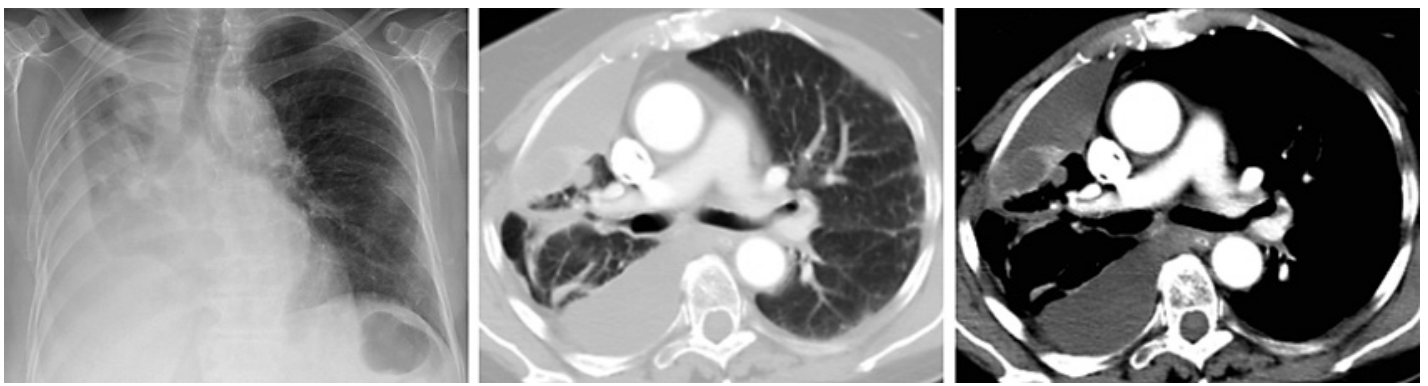

Fig. 6. Chest X-ray and CT after 4 months of treatment with osimertinib.

of 16 months, and during that time, RECIST v.1.1-assessed stable disease was achieved. The patient maintained an ECOG performance status of 1 with no significant adverse events.

In April 2017, the patient started complaining of chest pain with shortness of breath. A CT scan showed progression in terms of right pleural effusion, which was confirmed to be malignant by cytological examination (Fig. 5). A cytological examination of the pleural effusion revealed malignant cells (lung adenocarcinoma), and EGFR testing from the cytological sample was negative for any driver mutation. Bronchoscopy with TBB from the right upper lobe was repeated, and histology once more confirmed lung adenocarcinoma. EGFR testing confirmed the L858R mutation in exon 21. At that point, the tumor board recommended liquid biopsy (the cytology test from effusion was completely negative and the tissue EGFR positive for preexisting mutation). The liquid biopsy confirmed the L858R mutation in exon 21 and the T790M mutation in exon 20.

Karger's 
The patient was administered second-line therapy with osimertinib at a dose of $80 \mathrm{mg} /$ day. During the treatment, the patient showed no major adverse events, and the ECOG performance status was 1 . A CT scan after 4 months of therapy revealed a primary tumor in the right upper lobe, $4.7 \mathrm{~cm}$ in diameter with multiple bilateral pulmonary nodules, and right pleural effusion without lymph node enlargement (Fig. 6). According to the RECIST v.1.1 criteria, this was a partial radiological response.

Unfortunately, after 7 months of treatment, the patient experienced severe symptom worsening with deterioration of her performance status and symptomatic progression. The time to progression from the initial administration of osimertinib was 11 months and overall survival was 32 months.

\section{Discussion}

In this paper, we presented cases of patients with EGFR-positive stage IV lung adenocarcinoma who experienced progression to the first line of TKIs. It still remains debatable when it is strongly advisable to insist on tissue biopsy and when to initiate liquid biopsy and rely on its results. It is known that "tissue is always the issue," and this is a true statement in terms of histological confirmation of lung cancer types, immunohistochemical evaluation, and subclassification of lung cancer types. However, in the era of driver mutations and targeted therapies, this may not be the case. The issues of tumor heterogeneity, insufficient amounts of tissue needed for various tests, and patients' preference stress the need for a more universal, exact, amount-independent, and minimally invasive procedure. Liquid biopsy offers all of that and maybe more. Nowadays, besides single driver mutation tests, liquid biopsy offers the possibility of genome profiling, thereby providing insight into vast numbers of genetic alterations - in other words, opening the gates to many potential treatments of lung cancer. All patients in the case series had different scenarios of progression, and assessment of their mutation status was absolutely necessary for the decision on further treatment options. If this is unknown, if patients are misdiagnosed, or in case of false-negative results, patients' survival might be shortened by months. Two of the most important issues in biomarker testing, especially in terms of liquid biopsy, are timing and sequence. There is a limited amount of evidence-based data to support a clear definition of when to test a specific patient. In most cases, physicians must adhere to local recommendations, depending on availability and the reimbursement policy, which might not completely correspond to international guidelines.

In cases of EGFR mutation, it was proven in the AURA trial series that second-line osimertinib significantly improved survival outcomes. Without a meticulous approach to driver mutation detection, as well as case-by-case individualized diagnostics, many of the patients whose tumors harbor a T790M mutation might be misdiagnosed and mistreated [6-10].

In the phase I AURA trial, osimertinib was tested in 253 patients with advanced NSCLC who had radiologically progressive disease after treatment with a first-generation EGFR-TKI. In patients with a tumor biopsy-confirmed T790M mutation, osimertinib was reported to reach a response rate of $61 \%$ and a median progression-free survival of 9.6 months [6]. An analysis of phase I and II AURA trials reported equal efficacy of osimertinib independently of whether the T790M assessment was performed on plasma or on tissue from EGFR mutant patients with disease progression according to RECIST during first-generation EGFR-TKI treatment $[7,8]$.

Tumor biopsy should be performed on the primary or metastatic site during TKI therapy, not only to assess T790M but also to investigate any pathohistological transformation of the tumor [2]. Liquid biopsy can be an additional method, serving as a noninvasive alternative

\section{Karger'}


approach, in case tissue biopsy is impossible, there are contraindications, or tissue biopsy is rejected by the patient.

Hochmair et al. [2] published results from the largest real-world analysis of EGFR T790M prevalence following progression on afatinib in predominantly Caucasian patients with EGFR mutation-positive NSCLC. These results demonstrate that EGFR T790M mutation is the most common mechanism of acquired resistance (in $66.7 \%$ of cases). In their analysis, both liquid biopsy and tissue rebiopsy were used, with a concordance rate of approximately $80 \%$.

In 2 patients with acquired T790M mutation, osimertinib therapy was still ongoing, with exceptional overall survival. Our patient with primary T790M mutation reported a very good quality of life while being treated with the EGFR-TKI in first- and second-line therapy, and her symptoms were under control for more than 30 months.

There is no evidence, for now, that switching treatment according to T790M-positive ctDNA on liquid biopsy without disease progression according to RECIST could have an effect on therapy outcomes.

\section{Conclusions}

To achieve the best treatment results, it is imperative that NSCLC patients with EGFR mutation are screened for the EGFR T790M mutation at the point of acquired resistance. In this article, we showed a series of cases of NSCLC patients with tissue-negative mutation and ctDNA plasma-positive T790M and excellent treatment results with osimertinib as a secondline therapy after first-generation TKIs. Tissue biopsy yields the best results, but in some cases (poor location or size of the tumor at the time of disease progression, risks of complications that contraindicate rebiopsy, or patient disagreement with invasive procedures) liquid biopsy on the basis of ctDNA analysis can be trustworthy and a preferable noninvasive alternative approach.

A new challenge in the treatment of EGFR-positive NSCLC patients may be to better understand whether plasmatic progression occurs earlier than radiological progression according to RECIST. Future research will have to answer whether switching to next-line treatment solely on the basis of plasmatic progression could improve overall survival outcomes.

\section{Statement of Ethics}

Written informed consent was obtained from the patients for the publication of any related material. The investigational review board of the Institute for Pulmonary Diseases of Vojvodina, Sremska Kamenica, Serbia, with the ID No. 45/2020, provided their approval.

\section{Conflict of Interest Statement}

The authors have no conflicts of interest to declare.

\section{Funding Sources}

No funding was received for the publication of this study.

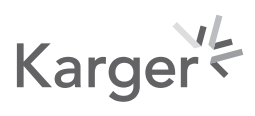




\section{Case Reports in Oncology}

Case Rep Oncol 2021;14:716-724

\section{Author Contributions}

All authors wrote the manuscript, performed the biopsies, and collected the data.

\section{References}

1 Yu HA, Arcila ME, Rekhtman N, Sima CS, Zakowski MF, Pao W, et al. Analysis of tumor specimens at the time of acquired resistance to EGFR-TKI therapy in 155 patients with EGFR-mutant lung cancers. Clin Cancer Res. 2013;19(8):2240-7.

2 Hochmair MJ, Buder A, Schwab S, Burghuber OC, Prosch H, Hilbe W, et al. Liquid-biopsy-based identification of EGFR T790M mutation-mediated resistance to afatinib treatment in patients with advanced EGFR mutationpositive NSCLC, and subsequent response to osimertinib. Target Oncol. 2019;14(1):75-83.

3 Ballard P, Yates JW, Yang Z, Kim DW, Yang JC, Cantarini M, et al. Preclinical comparison of osimertinib with other EGFR-TKIs in EGFR-mutant NSCLC brain metastases models, and early evidence of clinical brain metastases activity. Clin Cancer Res. 2016;22(20):5130-40.

4 Bosc C, Ferretti GR, Cadranel J, Audigier-Valette C, Besse B, Barlesi F, et al. Rebiopsy during disease progression in patients treated by TKI for oncogene-addicted NSCLC. Target Oncol. 2015;10(2):247-53.

5 Jänne PA, Yang JC, Kim DW, Planchard D, Ohe Y, Ramalingam SS, et al. AZD9291 in EGFR inhibitor-resistant non-small-cell lung cancer. N Engl J Med. 2015;372(18):1689-99.

6 Yang J, Ahn M, Ramalingam S. AZD9291 in pre-treated T790M positive advanced NSCLC: AURA study phase II extension cohort [abstract No. MINI16.06]. J Thorac Oncol. 2015;10:S319.

7 Mitsudomi T, Tsai CM, Shepherd F, Bazhenova L, Lee J, Chang GC. AZD9291 in pre-treated T790M positive advanced NSCLC: AURA2 phase II study. J Thorac Oncol. 2015;10:S320.

8 Lee CS, Sharma S, Miao E, Mensah C, Sullivan K, Seetharamu N. A comprehensive review of contemporary literature for epidermal growth factor receptor tyrosine kinase inhibitors in non-small cell lung cancer and their toxicity. Lung Cancer (Auckl). 2020;11:73-103.

9 de Mello RA, Neves NM, Tadokoro H, Amaral GA, Castelo-Branco P, Zia VAA. New target therapies in advanced non-small cell lung cancer: a review of the literature and future perspectives. J Clin Med. 2020;9(11):E3543.

10 Zarogoulidis P, Rapti A, Sardeli C, Chinelis P, Athanasiadou A, Paraskevaidou K, et al. Re-biopsy after relapse of targeted therapy. T790M after epidermal growth factor mutation, where and why based on a case series. Respir Med Case Rep. 2017;21:171-5. 